\title{
Categorical Perception of Color: Assessing the Role of Language
}

\author{
YASMINA JRAISSATI \\ Philosophy Department, \\ American University of Beirut
}

\begin{abstract}
Why do we draw the boundaries between "blue" and "green", where we do? One proposed answer to this question is that we categorize color the way we do because we perceive color categorically. Starting in the 1950's, the phenomenon of "categorical perception" $(C P)$ encouraged such a response. CP refers to the fact that adjacent color patches are more easily discriminated when they straddle a category boundary than when they belong to the same category. In this paper, I make three related claims. (1) Although what seems to guide discrimination performances seems to indeed be categorical information, the evidence in favor of the fact that categorical perception influences the way we perceive color is not convincing. (2) That CP offers a useful account of categorization is not obvious. While aiming at accounting for categorization, CP itself requires an account of categories. This being said, $C P$ remains an interesting phenomenon. Why and how is our discrimination behavior linked to our categories? It is suggested that linguistic labels determine CP through a naming strategy to which participants resort while discriminating colors. This paper's final point is (3) that the naming strategy account is not enough. Beyond category labels, what seems to guide discrimination performance is category structure.
\end{abstract}

Keywords: Color, categories, categorical perception, naming strategy, linguistic relativism

Why do we draw the boundaries between "blue" and "green", "purple" and "blue", "pink" and "red", etc., where we draw them? One possible answer to this question is that we draw the boundaries between categories where we draw them because we perceive color categorically. Starting in the 1950's a phenomenon was observed in speech perception and in perceptual categories that encouraged such a response. This phenomenon, called "categorical perception" (CP) effects, was canonically described by Harnad as follows: "Equal-sized physical differences be- 
tween stimuli are perceived as larger or smaller depending on whether the stimuli are in the same category or different ones" (Harnad, 1987, 3). Thus, CP effects, characterized by an enhanced discrimination performance, have been observed at category boundary. The existence of categorical perception has been postulated as an explanation of these effects, and consequently, categorical perception was put forward as an account of categorization.

However, the notion of "categorical perception" is problematic. Is categorical perception truly categorical? In other words, is the observed enhanced discrimination performance truly guided by categorical information or is it guided by perceptual cues? Also, is categorical perception truly perceptual? Or is it just that categorical information makes us better at judging that a given color sample belongs to a different category? In this case, however, categorical perception could not be said to be perceptual, and would rather result from a subsequent decision stage.

It is generally accepted that $\mathrm{CP}$ is categorical, and more specifically, that language determines $\mathrm{CP}$ in adults. The perceptual nature of CP effects is however harder to establish and this has important consequences on the account of color categorization that CP is supposed to offer. CP effects nevertheless remain interesting phenomena in need of explanation. Why and how are our color discrimination performances linked to our color categories? The most widely accepted account of CP is the naming strategy. It is thought that category labels guide discrimination (Kay \& Kempton, 1984). In order to decide which of the stimuli is the target in a cross-category discrimination task, participants are said to rely on the different labels they give to the different stimuli and discriminate on this basis. However, this account of $\mathrm{CP}$ is limited. It was shown that $\mathrm{CP}$ can be acquired without the use of language (Özgen \& Davies, 2002), and, more importantly, that CP can be observed within categories and not only at boundaries (Hanley \& Roberson, 2011). These results speak compellingly against the naming strategy account of CP.

In this paper, I would like to make three related claims. (1) Evidence that CP is truly a perceptual phenomenon is not convincing. (2) Although CP has been first introduced as an account of categorization, it is not obvious that it does offer such an account. Still, CP is intriguing. However, and this is my final point, (3) the most widespread account of this enhanced discrimination performance, i.e. the naming strategy, is not satisfactory.

\section{Is categorical perception, categorical perception?}

\subsection{The importance of perceptual cues}

Categorical perception effects refer to a phenomenon observed in perceptual categories: discrimination performance among adjacent samples is determined by category membership. This implies that the simi- 
larity experienced between samples is not entirely determined by their perceptual characteristics, or appearance, but by the category they belong to or do not belong to. This effect is also referred to as intercategory expansion. In fact, the CP phenomenon is seen as two-folded. Along with inter-category expansion, intra-category compression is also believed to occur (Franklin et al., 2005; Harnad, 1987; Özgen \& Davies, 2002). The latter refers to people being less sensitive to the perceptual difference between two adjacent colors when they belong to the same category. It was claimed in early works that items belonging to the same category could not be discriminated (Pisoni \& Tash, 1974). In their study, Studdert-Kennedy et al. even specify: "Subjects asked to discriminate between pairs of such 'categorical' stimuli are able to discriminate between stimuli drawn from different categories, but not between stimuli drawn from the same category. In other words, discrimination is limited by identification: subjects can only discriminate between stimuli that they identify differently" (Studdert-Kennedy et al., 1970, 234). This description of the phenomenon is however extreme, and a weaker form of CP is usually endorsed in subsequent color studies. According to this weaker form, which is of interest to us here, discrimination of neighboring colors within a category is possible but more difficult, thus slower and less accurate than discrimination of neighboring colors belonging to different categories.

Given that CP effects are measured by assessing discrimination performance, it is important to bear in mind the fact that perceptual cues do play a role. A better discrimination performance at a specific location, although consistent, does not obviously imply that it is not driven by perceptual cues. In order for categorical perception to be said to occur, the fact that what drives the decision in discrimination is categorical information rather than perceptual cues needs to be shown and cannot be taken for granted.

For this reason, several early studies focused on distinguishing between the two types of information. In same-different matching pair paradigms - where one sample is shown after the other and the participant asked to say whether the samples are the same or differentthe magnitude of the difference between the samples is varied, and performances are compared across magnitudes (Liberman et al., 1957; Pisoni \& Tash, 1974). Given that perceptual cues have some role to play in discrimination, more dissimilar stimuli are obviously discriminated more easily than more similar stimuli, regardless of whether or not they belong to the same category. The question behind these studies is therefore: At what point the physical information contained in the magnitude of the difference between samples is less or no longer useful for discrimination? And consequently, at what point does the categorical information become necessary? More generally, what this implies is that when arguing that participants rely on categorical information rather than perceptual features in discrimination tasks, the choice of the stimuli is crucial (Schouten et al., 2003). 
Perceptual cues can indeed produce a pop-out effect. Take the phenomenon of "linear separability" (Daoutis et al., 2006; Drivonikou et al., 2007), and three green color patches, G1, G2, G3, which are aligned and perceptually equidistant in a given Euclidean perceptual space. If, in a discrimination task, the target is either G1 or G3, the search is quick. However, if the target is G2, the search is slow. G1 can be separated from both G2 and G3 with a single straight line, it is therefore "linearly separable" (G1 | G2, G3), which makes it easy to discriminate. The same applies to G3, which is linearly separable from G2 and G1 (G1, G2 | G3). However, G2 cannot be separated by a single straight line from G1 and G3 (G1 | G2 | G3). Therefore it is not linearly separable. Thus, independently of the category to which they belong, color samples will pop-out depending on their linear separability, a perceptual feature. Clearly, if the target-distracter distance increases beyond a certain threshold, the search becomes effortless, regardless of the location of the target in the perceptual space in relation to the distracters.

Yet, several results clearly indicate that what guides discrimination performances is categorical information rather than perceptual cues. The most convincing evidence for the determining role of linguistic categories in categorical perception stems from cross-cultural comparisons. Cross-cultural comparisons are a straightforward way to establish that what guides discrimination is categorical information. If what produces a CP effect in a given observer is a language particular category, then, clearly, CP has to be guided by categorical information and cannot result from perceptual cues.

Several experimental paradigms designed to assess CP can be found in the literature, and are often used cross-culturally in order to compare CP effects at different language-dependent boundaries. One of the first such cross-cultural comparisons is Kay and Kempton's study (Kay \& Kempton, 1984) of Tarahumara color lexicon, where what could be considered a particular version of the triad, or odd-one-out task was used. In odd-one-out, participants are shown three psycho-physically equidistant stimuli simultaneously and asked to say which of the three is most different. Tarahumara speakers have one blue-and-green category instead of the two distinct English categories blue and green. Kay and Kempton found that English speakers consistently and accurately discriminated the 8 perceptually equidistant stimuli occurring in the 56 triads, along the blue/green boundary. Tarahumara speakers, who do not have two distinct categories for blue and green, could not discriminate as consistently. This shows that Tarahumara speakers do not experience the perceptual distance occurring between samples belonging to the blue and green English categories in the same way as English speakers do.

Other paradigms have also been used to measure CP: the visual search task, the two-alternative forced-choice memory task (2-AFC), and the same-different matching pairs. In the visual search task, a 
circle of several color patches (usually approximately 12) is presented on a screen (Gilbert et al., 2006; Zhou et al., 2010). All the patches are the same, except one, the target. The participant is asked to indicate whether the target is on the left or right half of the circle.

The 2-AFC is an adaptation of a paradigm widely used in the categorical perception of speech, the ABX paradigm (Liberman et al., 1957; Pisoni \& Tash, 1974). In ABX, the three stimuli A, B, and X are shown sequentially. Stimuli A and B are always different, $\mathrm{X}$ is always identical to either A or B. The participant is asked to say whether X is more similar to A or B. Given the constraints linked to speech events, the presentation of $\mathrm{A}, \mathrm{B}$, and $\mathrm{X}$ has to be sequential, as presenting two sound stimuli simultaneously is impossible (Roberson et al., 1999, 22). In color perception, samples can be presented simultaneously. Thus, in 2-AFC, instead of showing the three stimuli sequentially, the target $\mathrm{X}$ is shown first, individually, to the participant, then retrieved for a few seconds. Next, two stimuli, $\mathrm{A}$ and $\mathrm{B}$, are shown to the participant, simultaneously. The participant is asked to indicate which of $\mathrm{A}$ or B is most similar to the previously shown target. If both stimuli are from the same category, participants are slower and less accurate at identifying the target. However, if the two stimuli straddle a category boundary, participants are quicker and more accurate at identifying the target (see namely Roberson et al., 2000 and Roberson \& Davidoff, 2000 for the use of 2-AFC in cross-cultural studies of CP).

Finally, in the same-different matching pairs, participants are shown one sample. The sample is retrieved before another color sample is displayed and participants are asked to judge whether the samples they were shown are the same or different. When colors are from different categories they are judged as being different more quickly than when they belong to the same category, for an identical psychophysical difference (Bornstein \& Korda, 1984; Özgen \& Davies, 2002).

In 2-AFC and same-different matching tasks memory is clearly involved, as the participant needs to remember the target and compare it to the stimuli before responding. In the odd-one-out, used in the study involving Tarahumara described above, and in the visual search tasks, all color samples are presented simultaneously. Yet, in all four paradigms, color samples that do not belong to the same category are invariably more accurately and more quickly discriminated than color samples that belong to the same category, knowing that the perceptual distance between all used color samples is the same. The results from cross-cultural studies suggest in this way that categorical perception is indeed categorical.

\subsection{Categorical perception truly relies on categorical information}

The role of categorical information is also shown in the phenomenon of linear separability described above. Take for example the purple sample, in a blue, purple, pink triad. Purple is non-linearly separable 
from blue and pink given that it cannot be separated from blue and pink with a single straight line in the perceptual space. Still, unlike the triad featuring three green patches where the middle target does not pop-out, in the triad featuring blue, purple and pink, the purple sample is observed to pop-out. This result shows that when categorical information is available it facilitates the search, thereby reducing the effect of the perceptually driven linear separability. This result also points in the direction of the role of linguistic categories in discrimination (Drivonikou et al., 2007).

Thirdly, lateralization effect studies also show the role of categorical information in discrimination. More specifically, these studies aim at identifying the role of language in discrimination performances. As we have seen, in the visual search task paradigm, target and distracters are presented in a circle (Gilbert et al., 2006; Siok et al., 2009; Zhou et al., 2010). The distracters are all identical. Target and distracters are either from the same lexical category or from different categories, and the stimuli are all perceptually equidistant. Participants are asked to say whether the target, the color patch that is different from the rest, is in the right or left half of the circle.

Cross-category pairs are discriminated more easily than within-category pairs, however the results of these visual search tasks also show that in the cross-category pair, the discrimination is faster only when the target falls in the right visual field (RVF), not the left visual field (LVF) (Gilbert et al., 2006). To explain this discrepancy between LVF and RVF in discrimination speed, Gilbert et al. introduce a hypothetical connection with the language center found in the left hemisphere of the human brain. The RVF connects with the left hemisphere, whereas the LVF connects with the right hemisphere. Thus, the fact that the observers are fast at discriminating targets occurring in the RVF and not in the LVF is taken to show the influence of language on the cognitive representation of color. For targets occurring in the LVF, the categorical information would have to transfer from the left hemisphere into the right, across the corpus callosum, which is why discrimination in this visual field is slower. This advantage of the RVF is further supported by the discrimination performance of a callosal, split-brain, patient: no evidence of $\mathrm{CP}$ is found for targets occurring in his LVF (Gilbert et al., 2006).

Whether or not some discrimination also occurs in the LVF, and what it is taken to mean, is a subject of debate (Drivonikou et al., 2007; Roberson et al., 2007). If a CP effect, even fainter (Drivonikou et al., 2007), is also observed for targets in the LVF, the hypothesis according to which CP effects are constrained to the RVF because of its direct access to the left hemisphere, is weakened. Some, however, have argued that $\mathrm{CP}$ effects occurring in the LVF reflect innate universal categories rather than acquired ones, given that innate categories should be independent of the language center. 
The hypothesis according to which a universal categorization center, independent of language, is lateralized to the right hemisphere is supported by the results of visual search tasks studies involving prelinguistic infants. These studies show that $\mathrm{CP}$ effects in infants occur in the LVF, while it occurs in the RVF with adults. This suggests that there would indeed be innate categories, independent of language in infants, which would at a later stage be over-ridden by language-dependent boundaries (Franklin et al., 2008b). Still, other studies question the lateralization effect as a whole, as CP effects at language specific boundaries in Korean were found in both visual fields (Roberson et al., 2007).

Finally, the fact that $\mathrm{CP}$ is truly guided by categorical information in adults is shown in a fourth kind of study using neuro-imagery (Tan et al., 2008; Siok et al., 2009). These studies show that brain regions involved in language processes also take part in the neural networks that are activated during perceptual decisions in discrimination tasks. In their study, Tan et al. use a version of the same-different matching paradigm, featuring two samples on a uniform background, to which participants need to respond by "same" or "different" (Tan et al., 2008). In one condition, the stimuli are easy to name, non-ambiguous colors. In the other condition, the stimuli are difficult to name, ambiguous colors. Participants are equally accurate and quick at discriminating colors in both conditions. The results indicate that apart from the regions that are activated in both conditions, the easy-to-name condition also recruits the left superior temporal gyrus. Given that these circuits subserve word-finding processes, Tan et al. conclude: "language appears to affect neural activity patterns activated in the course of color perception" (Tan et al., 2008, 4007)

Wanting to see more specifically if these areas of the brain are more readily recruited when the targets are shown in the RVF rather than in the LVF, Siok et al. proceed with a study involving both a visual search task and fMRI, where participants' brain activity is scanned during discrimination (Siok et al., 2009). Their results show that the levels of activity in the left temporoparietal areas (BA 40), responsible for language processes, and areas crucial in color vision (V2/3) are both significantly intensified when stimuli from different categories are presented in the RVF. The author's tentative conclusion is that "the posterior temporoparietal cortex serves as a top-down control source that interacts with and modulates the activity of the visual cortex (V2/3)" (Siok et al., 2009, 8143).

\subsection{Categorical perception is truly perceptual}

Thus, an important set of results strongly suggests that what guides discrimination performance in $\mathrm{CP}$ is categorical information. However, is categorical perception truly perceptual? For CP to be categorical perception, categorical information should influence perception and 
perceptual experience, not a subsequent decision stage. If categorical information does not influence perception, but merely reflects an improvement in judgment of perceptual differences, then $\mathrm{CP}$ cannot be said to be perceptual.

The locus of the effect is critical, and this question was initially addressed in terms of the kind of information processing involved. Either the information is processed in a serial way, first involving lower perceptual stages and then higher cognitive ones (and consequently slower and non automatic); or the information is processed in parallel, which implies that physical and categorical information are processed at the same time. Consequently, parallel processing is fast, automatic and effortless (Rensink \& Enns, 1995). Thus, the predicted processing times should differ in a serial or a parallel model (Wood, 1974; Liberman et al., 1957; Pisoni \& Tash, 1974; Bornstein \& Korda, 1984). According to Bornstein and Korda (Bornstein \& Korda, 1984), in a serial model, physical information about the stimulus is processed before categorical information. As a consequence, the categorical information contained in the straddling of a category boundary, and which is processed at a later stage, should not reduce the RT in a "different" condition where the two stimuli being discriminated belong to different categories. In a parallel model however, physical and categorical information are processed at the same time. The categorical information consisting in the fact that the two stimuli belong to different categories is redundant with the physical information and should facilitate the "different" response, thereby reducing the RT.

Bornstein and Korda proceed with two kinds of matching tasks, a "physical match" condition, where participants are asked to match the two stimuli according to their physical resemblance, and a "categorical match" condition where participants are asked to match the two stimuli according to the categories to which they belong. In the physical match condition, participants need to attend to the physical information only. In the categorical match however, participants need to use both physical and categorical information. If observers process physical and categorical sources of information in parallel, category boundary should facilitate the discrimination in "different" trials, where the two stimuli belong to different categories. Furthermore, they should also be faster in "same" trials, where the stimuli belong to the same category, under the categorical match condition as well. The reasoning behind this last prediction is that participants will judge that the two stimuli belong to the same category, and therefore be quicker at responding "same".

Bornstein and Korda's results confirm their predictions. Redundant information, physical and categorical, decreases reaction times, and this fits with the parallel processing model, not the serial one, according to Bornstein and Korda. They therefore conclude that categorical information is available very early in information processing. "The re- 
sults point to a role of cognitive (i.e., categorical) information in otherwise purely sensory (i.e., psychophysical) assessments" (Bornstein \& Korda, 1984, 221). Such conclusions are also reached in studies involving categorical perception of speech (Wood, 1974; Liberman et al., 1957; Pisoni \& Tash, 1974, but see Pilling et al., 2003).

\section{Reconsidering the role of language}

\subsection{Categorical perception may not be perceptual after all}

In answer to the question "does CP rely on perceptual cues or categorical information?" four different kinds of studies strongly suggest that categorical perception is indeed categorical. In the process of showing that categorical information is relied on, a more definite claim is put forward. Namely: it is not just categorical information that determines discrimination performance in adults, but linguistic codes, specifically. Cross-cultural studies, linear separability, lateralization effects, and fMRI studies, all imply that what underpins CP in adults is language and lexical categories. Although the fact that language intervenes in CP seems to have been clearly shown, what exactly the role of language consists in is still hypothetical.

In one of the first studies looking into adult cross-cultural CP of color (Kay \& Kempton, 1984), Kay and Kempton suggest that the role of language amounts to a naming strategy to which participants resort during discrimination:

A triad like (A, B, C) presented a difficult judgment to make. When one is shown the triad (A, B, C), it is obvious only that B is not the most different. We propose that faced with this situation the English speaking subject reasons unconsciously as follows: 'It's hard to decide here which looks the most different from B, A or C. Are there any other kinds of clues I might use? $\mathrm{Aha}$ ! $\mathrm{A}$ and $\mathrm{B}$ are both CALLED green while $\mathrm{C}$ is CALLED blue. That solves my problem; I'll pick C as most different.' (Kay \& Kempton, 1984, 72).

The idea that the role of language in color CP specifically consists in a naming strategy is subsequently found in several other studies (Franklin \& Davies, 2004; Özgen \& Davies, 2002; Regier et al., 2010; Roberson et al., 2007). However, what the presence of such a strategy really means when it comes to the perceptual nature of the phenomenon is debatable. That it is not entirely desirable for language to play a straightforward role in CP is often disregarded. In fact, the naming strategy approach is double-edged. On one hand, looking into the involvement of language specific networks during discrimination tasks serves to show that the kind of information required to discriminate is categorical, not perceptual, which is the essence of CP. On the other, if the role of language were too straightforward, this would mean that participants are merely naming the colors they discriminate. Or, to put it in Winawer et al.'s words, that they "rely only on linguistic representations" (Winawer et al., 2007, 7780), which contradicts the idea of a warping of the perceptual space supposed to take place in CP (Özgen 
\& Davies, 2002; Schouten et al., 2003; Daoutis et al., 2006; Regier et al., 2010). Indeed, for CP to be perceptual, discrimination, although guided by categorical information, should nevertheless imperatively take place at a low perceptual level.

To disambiguate the role of language, a few studies featured discrimination tasks and verbal interference tasks executed in parallel (Roberson \& Davidoff, 2000; Winawer et al., 2007). An interference task is one that is meant to disrupt the discrimination. If the interference task is verbal, this means that the disruption involves language specific capacities. If the interference task is visual, the disruption involves perceptual capacities. A first notable study to have used verbal interference task as a way to determine the role of language in CP is Roberson and Davidoff's (Roberson \& Davidoff, 2000). In their study, Roberson and Davidoff use a 2 -AFC task using colors and facial expressions. The verbal interference tasks varies from presenting words on screen during the 5 or 10 seconds interval between presentation of target $\mathrm{X}$ and presentation of stimuli $\mathrm{A}$ and $\mathrm{B}$, to having participants repeat a string of numbers backwards and out loud. Their results show that only the verbal interference task affects cross-category discrimination, not visual interference tasks. Although visual codes must clearly play a role in both kinds of discriminations (within- and cross-category), the fact that verbal interference makes CP effects disappear suggests the importance of linguistic codes in cross-category discrimination.

In another study using verbal interference in color discrimination, Winawer et al. use not a 2 -AFC task, which relies on memory, but an odd-one-out task, where the target and distracters are all simultaneously present on screen (Winawer et al., 2007). The tested boundary is the one occurring between the Russian siniy and goluboy categories (dark and light blue respectively). Participants discriminate in three conditions: no interference, verbal interference, and visual interference. In the visual interference condition, before the stimuli set is displayed, participants are presented with a pattern, a grid of white and black squares. After the discrimination, they are shown two grids, and asked which of two grids is the one they were shown before the discrimination task. In the verbal interference condition, participants are presented with a string of numbers before the stimuli set is displayed. After the discrimination task, they are shown two strings of numbers and asked which was the one they were previously shown. Both interference tasks therefore require participants to remember either a visual pattern or a string of numbers. In the case of the string of numbers, in order to remember it, participants are asked to repeat it silently to themselves while discriminating.

The first important result of this study is that, as expected, in the no interference condition, $\mathrm{CP}$ is observed at this language specific boundary with native Russian speakers, but not with English speaking Americans. This suggests, again, that discrimination is guided 
by categorical information. Second, as in the Roberson and Davidoff study, the cross-category discrimination performance is affected by the interference tasks, but only by the ones that are verbal. Thus, despite the simultaneous presence of the target and distracters on screen, despite the fact that participants are not required to commit the target to memory, or to store a linguistic code in order to discriminate, verbal interference disrupts discrimination performance. The fact that only the verbal interference task affects discrimination implies that language plays an essential role in CP. More specifically, Winawer et al. suggest that an online access to language is required in order for $\mathrm{CP}$ to occur.

However, if verbal interference disrupts CP, then it cannot be said that language leads to permanent changes in perceptual processors. As Winawer et al. rightly point out, literature in perceptual learning has shown that very specific improvements in perceptual performance are possible, supposedly reflecting a corresponding change in synaptic connections in early stages of the visual processing (Karni \& Sagi, 1993). However, this is not the kind of influence language can be said to have on color categorization. If a linguistic code needs to be accessed in order for CP to occur, the extent to which this effect is perceptual is not obvious.

\subsection{CP cannot account for categorization}

As Harnad explains is his widely cited paper (Harnad, 1987), the idea underlying categorical perception is to offer a uniform account of categorization. Why do we organize a physically continuous stimulus into discrete entities? One possible answer to this question is: we organize color in discrete entities because we perceive it categorically. We have just seen that it is not obvious that, in adults, categorical perception is truly perceptual. This would mean that we do not perceive categorically.

If "categorical perception" is not perceptual, then what it is? Is it just a "categorical partitioning" as some have suggested (Massaro, 1987), and its effect just a "categorical effect" (Davies \& Corbett, 1998; Drivonikou et al., 2007; Daoutis et al., 2006)? If categorical perception is rather "categorical partitioning" and simply refers to the fact that we partition the color space into categories, how is it different from categorization? Thus, if categorical perception is not perceptual, then it cannot offer an account of categorization.

Even if we were to admit that CP was perceptual, it is still not clear how the notion can account for categorization. It is suggested that we draw the boundaries between categories because we perceive categorically. But why, then, do we perceive categorically? Introducing the notion of "categorical perception" merely postpones the problem, but does not solve it.

Nevertheless, an important part of the research on categorical perception of color focuses on answering the question why we perceive 
categorically. Suggested answers vary from categorical perception is acquired, to $\mathrm{CP}$ is innate, or, most plausibly, to $\mathrm{CP}$ is both innate and acquired. In support of the acquired nature of categorical perception, results from cross-cultural comparisons developed in 1.1 are put forward. If CP varies across languages and is observed at language-specific boundaries, then CP effects cannot be innate and has to be acquired through language.

However, studies on pre-linguistic infant color categorization contradict this claim to some extent (Bornstein et al., 1976; Franklin \& Davies, 2004; Franklin et al., 2005; Franklin et al., 2008; Franklin et al., 2008). If CP is determined by language, then it should not be observed in infants who have not yet acquired language. If, however, CP is observed in pre-linguistic infants, then it cannot be said that $\mathrm{CP}$ is exclusively determined by language. In a pioneering study using novelty preference as an indicator of $\mathrm{CP}$, Bornstein tests 4-months-old infants. This technique relies on target looking time or gaze direction. Infants are habituated to a target by being exposed to it for a certain amount of time. They are then shown another target that varies from the first. The novelty preference measure implies that infants will look longer at a target they perceive as new, in comparison to the stimulus to which they were habituated. Thus, if infants look long at the second stimulus, it is inferred that they perceive the stimulus as being different from the first. If however, they do not look long at the second stimulus it is inferred that infants perceive it to be the same as the first. Bornstein tests the infants with monochromatic light stimuli. Infants respond differently at wavelengths falling into different adult categories (for example blue at $480 \mathrm{~nm}$ and green at $510 \mathrm{~nm}$, separated by a magnitude of $30 \mathrm{~nm}$ ), than they do to wavelengths separated by the same magnitude, but falling in the same category (for example $450 \mathrm{~nm}$ and $480 \mathrm{~nm}$ which are both categorized as blue by adults and are also $30 \mathrm{~nm}$ apart). Bornstein in this way detects boundaries between blue and green, and red and yellow (Bornstein et al., 1976). These results suggest that prelinguistic infants experience CP at category boundaries, and therefore speak against a language-dependent view of categorical perception.

In a replication of this study, Franklin and Davies tested 36 4-months-old infants, using Munsell physical samples instead on monochromatic lights, and a video-recording of infants' reactions and direction of gaze (Franklin \& Davies, 2004). In their experiment, the novel stimulus is presented alongside the stimulus to which the infant was habituated. They observed a CP effect at the blue/green boundary, but also at so-called secondary boundaries blue/purple and red/pink — purple and pink are considered secondary categories (Hering, 1964; Kay \& McDaniel, 1978). Franklin et al. also test toddlers in the process of color lexicon acquisition, cross-culturally (Franklin et al., 2005). In this study, they use a 2-AFC task featuring bear toys. Finally, Franklin et al. test toddlers using a version of the same-different matching 
task, where one stimulus, instead of being shown after the other as is usually done, is shown on a colored background (Franklin et al., 2008a; Franklin et al., 2008b). The results of all these studies are consistent with previous findings: young children manifest CP at language-independent category boundaries.

It is worth noting that studies involving infants and toddlers have been criticized from a methodological perspective. A replication of the Franklin and Davies 2004 study (Franklin \& Davies, 2004) was found to be impossible, as 4-months-old participants seemed to have found the task uninteresting, and were restless (Davidoff et al., 2009). Second, no direct comparison with adults is possible because different color sets are used, given that the ones used with adults are too difficult for infants to discriminate (Franklin \& Davies, 2004; Davidoff et al., 2009). Davidoff et al. also note that for the data gathered with infants to be truly a confirmation of the innateness of category boundaries hypothesis, boundaries that are considered non innate, such as the Russian siniy/goluboy boundary should also be tested with infants, who should not manifest it (Davidoff et al., 2009).

Regardless of how reliable studies involving infants are, even if one was to admit the results of pre-linguistic infant color categorization, what they show is that CP may be innate, not that it cannot be acquired. The fact that infant and adult $\mathrm{CP}$ does not always occur at the same boundaries suggests that even if some CP is innate, it obviously does not affect adult color discrimination. The latter still seems to be influenced by linguistic categories. Franklin et al.'s suggestion is that color categories are hardwired, probably involving cortical structures, beyond the visual cortex, and that they are later tuned by the environment (Franklin \& Davies, 2004). This line of thought offers that innate $\mathrm{CP}$ would guide the formation of categories in young children, and that once these categories are formed based on both perceptual and linguistic or cultural factors, they in turn determine CP in adults (Franklin et al., 2008b). Such a conclusion is not in contradiction with the Whorfian interpretation of the cross-cultural studies' results. To the extent that speakers of different languages experience $\mathrm{CP}$ at different boundaries that correspond to their lexical categories, the influence of language on perception cannot be denied. Furthermore, to the extent that the boundaries identified in pre-linguistic infants are not manifest in adults cross-culturally, such innate boundaries are clearly over-ridden by linguistic ones. Therefore, the observation of CP in prelinguistic infants, if it were to be admitted, would be relevant for our understanding of what drives the formation of color categories in the first place. It has however less impact on the question pertaining to the influence of language and lexical categories on perception.

Although some answers to the question why we perceive categorically have been offered, it does not change anything to the fact that the notion of categorical perception cannot account for categorization. In 
order for CP to explain why adults perceive categorically, an account of the nature of the categorical information is needed; or in other words, an account of categories is required. The use of categorical perception as an account of categorization seems therefore circular.

More generally, what CP truly amounts to strikes us as a legitimate worry. A distinction should be made between so-called "categorical perception effects", that refer to non-monotonous discrimination performances observed in relation to categories, from "categorical perception" supposed to account for such effects. Some researchers show that a non-categorical approach to perception, or a continuous approach to perception, accounts equally well, and in some cases even better, for such a behavior (Massaro \& Cohen, 1983; Massaro, 1987). Why then, should we posit the existence of $\mathrm{CP}$, if our discrimination performance can be accounted for without it? On their view, there is no need to talk of categorical perception. It is not perception that is categorical, but quite simply, the subsequent decision stage. That is not to say that research on categorical perception is pointless. To the contrary, it has greatly contributed to our understanding of color cognition. Moreover, categorical perception, or the enhanced discrimination performance linked to category possession, remains an interesting phenomenon in and of itself. And the question of the nature of the categorical information guiding discrimination performance is still relevant.

Indeed, we have seen that the most widely accepted account of categorical perception in adults introduces a naming strategy. The naming strategy account suggests that adults discriminate based on their lexicon. Categorical perception would therefore be guided by differences in color labels. In the last part of this paper, I argue that such an account of enhanced discrimination performance is not enough. More specifically, the naming strategy cannot account for all cases of CP. There is more to our discrimination behavior than labels.

\subsection{CP acquisition does not necessarily involve color labels}

The possible irrelevance of a naming strategy in CP is first indirectly shown in a study involving CP acquisition by Özgen and Davies (Özgen \& Davies, 2002). The linguistic account of CP, according to which language underpins adults' experienced color discontinuity, versus innate boundaries, presupposes that CP can be learned. Indeed, if CP is not innate but occurs at language specific boundaries, then $\mathrm{CP}$ must have been acquired. In order to tackle the specific question of whether or not CP can be acquired, Özgen and Davies train 40 participants on a new boundary. The new boundary on which participants are trained is set on the existing focal point of green or blue. In other words, the focal point of blue is at the boundary of the first new category, and the focal point of green at the boundary of the second. The focal point of the new category is therefore at the previous boundary between blue and green. After training on the new boundary, participants are tested 
using a same-different judgment task on both blue and green samples. The target is displayed for $500 \mathrm{~ms}$, retrieved for $500 \mathrm{~ms}$, and followed by another stimulus that the participant has to judge as being the same or different from the previously shown target. Control groups who haven't undergone the training also take the test.

The first result is an increased accuracy in the trained region in both trained groups: Both groups perform significantly better than the controls on the boundary they have newly acquired. In other words, for the region on which they are trained, participants manifest $\mathrm{CP}$ at the new boundary, disregarding the old focal point and overriding previous $\mathrm{CP}$. While CP remains the same in the region in which they are not trained. Thus, CP obviously seems to have been acquired.

Özgen and Davies had predicted that if such categories could be learned, "such a finding would support the possibility that that crosslanguage differences in behavior on color tasks similarly arise as a result of the influence of learning linguistic categories on perception" (Özgen \& Davies, 2002, p.478). However, it should be noted that no language was used during the training. In fact, Özgen and Davies design the experiment so as not to favor a naming strategy and avoid a criticism of the kind described in 2.1. They make it a point that no category labels are available to participants. Participants learn the categories in a contextual manner, based on feedback, and at no point are labels given to the new categories.

Participants are trained over three consecutive days. They first undergo a context training phase in either the "new green $1 /$ new green2" condition, or in the "new blue1/new blue2" condition. In this context, they are presented with a screen with eight slots to the left and eight slots to the right and asked to drag and drop the incoming individual colors into one of the slots to the left or right, depending on if they belong to the same category or not. They are instructed to "successfully learn the rule that separates these two groups" (Özgen \& Davies, 2002, 482). No other indication as to how to categorize the colors is given, nor information regarding what the categories are based on. Participants receive immediate feedback. If they categorize correctly, the color remains in the slot. If not, the color disappears and a sound is emitted. The stimuli they judge correctly remain on screen during category judgment. In the second stage of the training, one color at a time appears on the screen, and participants have to say whether the color goes to the left, or to the right, based on what they learned in the previous training phase. They are again given immediate feedback.

Thus, given how categories are learned, it is clear that at no point are labels involved. Özgen and Davies even specify: "There were no category labels available to the participants, and the only labeling could have involved labeling each individual stimulus in the set" (Özgen \& Davies, 2002, 492). In these conditions, it can hardly be argued that participants discriminate on the basis of their language. This study 
aimed at showing that $\mathrm{CP}$ can be acquired. It successfully does so. Nevertheless, what this study also shows is that CP can be acquired without the use of labels, as what is observed is the influence of an acquired category, however not a linguistic one.

\subsection{CP is observed within categories as much as at category boundary}

In a recent work, Hanley and Roberson go over 7 previous studies of CP that used 2-AFC, mostly in color discrimination but also facial expressions and facial identity (Hanley \& Roberson, 2011). They observe a pattern in the results that had not been previously detected. Invariably, and as we have seen in other studies, adjacent patches of color that belong to different categories are discriminated more easily than adjacent patches of color that, although featuring the same perceptual distance between the stimuli, belong to the same category. Unsurprisingly, all participants perform significantly better on the pair that straddles the category boundary, than on the pair that doesn't.

In their study, Hanley and Roberson however show that CP also occurs within categories if in the within-category pair the target is a better example of the category than the distracter. More specifically, participants are as quick at discriminating a "good example" target in a within-category pair, than they are at discriminating colors in a cross-category pair. It is only when the target is a "bad example" of the category that the discrimination is slower in a within-category condition than in a cross-category condition. This applies to all seven studies re-assessed by Hanley and Roberson.

What this result straightforwardly implies is that linguistic information as articulated in a naming strategy is not enough to account for $\mathrm{CP}$. The fact that within-category pairs are discriminated as well as cross-category pairs when the target is a good example of the category suggests that participants cannot be going through a process along the lines of "the target is blue, distracter $\mathrm{A}$ is blue, but distracter $\mathrm{B}$ is green, therefore B is most dissimilar from the target", since in this case, both distracters, A and B belong to the same category and are both labeled "blue", or both labeled "green". What seems to guide them in their decision has to do with the category's inner structure. It is the degree at which a given sample is labeled green or blue that makes a difference in the discrimination performance. This kind of judgment of membership supposes a representation of the category's structure, with its central prototype and less clear members on the edges, and cannot be explained on the basis of pure labeling.

For the series of studies re-assessed by Hanley and Roberson, and for all the other studies having taken place in the same vein, this result is problematic. It falsifies the naming strategy account of $\mathrm{CP}$, which underpins the cross-linguistic studies, as well as the lateralization and fMRI studies. If linguistic labels are not necessary for CP to occur, and given that $\mathrm{CP}$ experienced by adults is not innate, can there be some- 
thing else that guides participants' discrimination? Even if linguistically acquired categories do cause $\mathrm{CP}$, given that categories acquired independently of language also cause $\mathrm{CP}$, it can no longer be implied that what causes CP is necessarily category labels. It may well be that categories' inner structure is at least as important as labels in CP. Or even that in $\mathrm{CP}$, category labels in fact latch onto something else, such as category structure.

\section{The role of category structure}

\subsection{The role of category structure as it was previously observed}

Clearly, given the uncontroversial results from cross-cultural studies, it is difficult to deny that language plays a role in CP. What should perhaps be revised in previous interpretations of discrimination performances is the role language is proposed to play in CP. We have just seen that a naming strategy, although a favored approach, is doubtful in light of some results.

Hanely and Roberson's research suggests that what seems to be crucial in CP effects is color category structure, not color lexicon. Another result, reported by Bornstein and Korda, also points in the same direction. In their study, Bornstein and Korda (Bornstein \& Korda, 1984) examine the kind of information influencing participants' discrimination performance: physical information, categorical information, or both.

Bornstein and Korda use a same-different matching task, where two stimuli are shown one after the other. They are either from the same category or from different categories. When they are from the same category, they are either identical, or different. Bornstein and Korda ask participants to say, based on categorical information (not color appearance), whether the stimuli are the same or different. Unsurprisingly, in the "same" condition, there is a significant difference in RT between pairs of stimuli that are identical, and pairs of stimuli that are different. Participants are faster at judging that the stimuli are from the same category when they are identical, and slower at judging that the stimuli are from the same category when they are different.

In the "different" condition, where stimuli straddle the blue/green category boundary, participants are slow at judging that stimuli are different, when 2 Munsell $^{1 *}$ hue steps separate the stimuli, at constant lightness and saturation levels. They are significantly faster when

1* The Munsell model is a representation of our color experience developed by Albert Munsell in the beginning of the 20th century. The spheroid color solid features three dimensions: lightness, which varies from black, at the bottom of the central axis, to white, at the top; hue, which ranges from red, to orange, yellow, green, blue, purple and red again on a circle at the center of which passes the lightness axis; saturation which represents the intensity of the color, and is the dimension lying between the central lightness axis and the outer skin of the solid. The model relies on equal perceptual distances between adjacent color samples, in the three dimensions of hue, lightness and saturation. 
there are 4 or 6 Munsell hue steps between the pair of stimuli straddling the boundary. This expected result suggests that "different" responses to stimuli belonging to different categories can be made solely on the basis of physical information.

However, according to Bornstein and Korda, "same" judgments are not made on the basis of physical information only. Indeed, when the pair of stimuli is closer to the category center, the reaction times are quicker. Conversely, when the pair of stimuli is closer to the boundary, the reaction times are slower. If "same" judgments were made only on the basis of appearance, equidistant stimuli pairs would yield similar RTs, regardless of their distance from the category center.

This pattern of results reflects participants' performance in the category identification task. In the identification task, participants are asked to say if the sample they are seeing is blue or green. The closer the sample is to the center of the category, the quicker the response. The closer the sample is to the category boundary, the slower the response. The identification task helps in assessing the category extension and the category boundary at which CP effects are observed. The fact that performance in the same-different matching tasks with stimuli taken from within the category correlates with the performance in the identification task implies for Bornstein and Korda that both categorical and physical information are simultaneously involved, in parallel, in observers' decisions during discrimination. If discrimination only relied on perceptual cues, then equidistant pairs of stimuli would be equally slowly discriminated regardless of how close the pair is from the category center. This is how Bornstein and Korda argue that (1) the cues on which discrimination relies are not only perceptual but also categorical, and (2) something closer to a parallel processing model than to a serial processing model is taking place in CP.

Thus, Bornstein and Korda observe that discrimination of colors is non monotonous not only at category boundary, but also within category. In this case as well, an implicit naming strategy, where participants use the available green and blue labels to discriminate, is not relevant. A pair of samples will be judged to be the same more quickly than another if it is closer to the center, or if it is a good example of the category, even if both pairs carry the same "green" or "blue" label.

\subsection{The importance of correlating category identification and CP}

Bornstein and Korda and Hanley and Roberson's studies draw attention to the fact that it is not enough to assess the location of the linguistic boundary and to test for $\mathrm{CP}$ at the boundary to have a complete picture of what seems to go on in the observer's mind. The fact that the category also has an internal structure plays a role, and this structure is not directly linked with language.

The graded nature of color categories is not a newly observed feature (see namely Rosch, 1973; Kay \& McDaniel, 1978), but somehow, this 
important aspect of color categories has been put aside in CP studies. Yet, early studies insisted on the correlation between identification and $\mathrm{CP}$ effects. It was stressed on several occasions that a better discrimination performance at category boundary meant categorical perception only if this discrimination performance correlates with category identification (Pisoni \& Tash, 1974; Liberman et al., 1957; Roberson et al., 1999; for more on this issue see also Schouten et al., 2003). Category identification performance is characterized with faster identification in the center, and slower identification at the boundaries, where the CP effect is precisely expected to occur. It was even suggested that "discrimination could be predicted on the basis of the identification probabilities alone" (Pisoni \& Tash, 1974, 285), implying that CP is expected to occur where category membership is less certain.

The fact that current studies of CP focus on inter-category expansion, disregarding category internal structure, is most obvious in studies such as the one by Zhou et al. (Zhou et al., 2010). This study, which features both training on new categories and fMRI, is taken to show both that $\mathrm{CP}$ can be acquired via language, and that newly acquired $\mathrm{CP}$ recruits brain areas involved with language.

Like Özgen and Davies, Zhou et al. also train participants on new categories. In this case however, each new category consists of a single sample on which participants are trained and tested. The set of four categories are neighboring samples in the blue/green area. Each new category is given an arbitrary name. Participants are trained on three consecutive days using a listening, a matching, and a naming task. On the fourth day they are tested for CP using a visual search task, where LVF and RVF can be distinguished. A control group is also trained in the same way, except that no new names are introduced in this case, and their training involves the names participants already know for green and blue. They also take the visual search task test on the fourth day.

Results indicate that $\mathrm{CP}$ is acquired at the boundaries of the new categories. The fact that the control group that was exposed to the same colors the same amount of time does not manifest CP at new boundaries, but at their own blue/green boundary, is taken to suggest for Zhou et al. that what is observed with the trained participants is indeed CP at novel boundaries, and not just an enhanced discrimination behavior induced by repeated exposure to a limited number of stimuli.

The fact that the controlled variable is category labels shows that Zhou et al. presuppose that language is required for CP to occur. This interpretation of the result contradicts Özgen and Davies' finding which shows that CP can occur in the absence of labels. In Zhou et al., the importance given to labels obscures the role of category structure to the point that the novel categories consist each of a single sample. Such an experimental setup makes no room for the observation of color identification performance, which is characterized by consistent or fast 
responses for the clear members at the center and less consistent or slow responses for ambiguous members at the boundaries. If CP were to be interpreted strictly, as is encouraged by some (Schouten et al., 2003), and a correlation had to be observed between identification and discrimination, it is not clear that Zhou et al.'s results can be taken to show CP. What we observe is a better discrimination performance between samples that carry different labels, and the activation of language networks in the brain during discrimination. However, because unlike in other studies there is no minimal correlation with an identification performance, these results cannot be taken to mean anything else apart from the fact that participants get better at discriminating between samples, using a naming strategy.

Nothing, apart from the discrimination performance, indicates that participants have indeed acquired categories. Some have suggested CP might be a definition of category membership (Roberson et al., 1999). However, relying on discrimination performance to suggest that categories have been acquired is in this case circular: the fact that what is observed is indeed CP presupposes the existence of a category; and yet the existence of a category is taken to be shown by the observation of an improved discrimination performance.

If the fact that a category is indeed acquired is called into doubt, Zhou et al.'s results can be used to support a claim opposite to the one they have been putting forward. These results can indeed be taken to show that a better discrimination performance can follow from training independently of category acquisition. This may further show that label acquisition is not necessarily accompanied by category acquisition. In fact, the converse of this last conclusion, namely that category acquisition is not necessarily accompanied by labeling, is what we take Özgen and Davies' study to show (Özgen \& Davies, 2002). The point here is not to say that labels and categories are independent from each other, but to show how the lack of correlation between discrimination performance and category identification and structure affects our understanding of CP.

\subsection{The possible role of category labels}

In their study, Hanley and Roberson offer an alternative account for the role of language in $\mathrm{CP}$, building on one further result. Indeed, in their study Hanley and Roberson also look at the effect of verbal interference on within-category discrimination (Hanley \& Roberson, 2011). We have seen that verbal interference tasks destroy CP effects at category boundary. The explanation of the disruption caused by verbal interference tasks is that participants are not able to access their lexicon while discriminating and are therefore unable to use a naming strategy. After having shown that $\mathrm{CP}$ also occurs within categories when the target is a good example, Hanley and Roberson examine the effect of verbal interference on within-category discrimination. They observe 
that verbal interference also disrupts within-category discrimination. Except that, in the case of within-category discrimination, verbal interference improves the performance when the target is a poor example of the category. This result is not observed as a result of verbal interference in cross-category conditions.

Hanley and Roberson's account of this phenomenon rests on Pisoni and Tash' suggestion that category labels or codes are most effective in $\mathrm{CP}$ when the target is a good example of the category (Pisoni \& Tash, 1974). Hanley and Roberson note that in 2-AFC, since perceptual information is not available during discrimination, participants rely on the category code. Thus, how well they encode the target's color at presentation will affect how well they discriminate between the two stimuli. If the target is a boundary color, the encoding may not be consistent. Therefore, when the target is a poor example of the category, the linguistic code is not as reliable as when the target is a good example during discrimination, resulting in higher RTs. Furthermore, colors are sensitive to context. Based on this sensitivity, Hanley and Roberson suggest, that when presented in the context of good examples, a poor example is likely to be named differently. If this is true, then the fact that verbal interference improves discrimination performance in within-category conditions when the target is a poor example is explained by the fact that verbal interference disrupts this context effect. The poor example is no longer given the name of a different category, and discrimination is quicker.

If this hypothesis were to be verified, this would offer an alternative interpretation of the role of labels in CP. It is not, therefore, that participants would resort to a naming strategy applied to colors found on either side of a lexical boundary. Instead, participants' naming of colors would depend on how good an example of a given category a given color is. In any case, what this alternative interpretation of the role of language implies is that language alone, in the sense of linguistic labels or codes, is not enough to account for CP. It has to be accompanied by a representation of the category's internal structure (for more on category structure see Jraissati et al., 2012).

\section{Conclusion}

Categorical perception is a phenomenon that is widely studied in the color cognition literature. The notion was initially introduced to answer the question of categorization. It was suggested that we categorize color the way we do because we perceive it categorically. I have argued that the notion of $\mathrm{CP}$ is however problematic. First, it is not clear that CP is indeed perceptual. Consequently, and if categorical perception is not perceptual, then all that CP means is that we categorically partition the space, or that we categorize. In other words, if it is not perceptual, CP cannot offer an interesting account of categorization. Furthermore, even if the perceptual nature of $\mathrm{CP}$ was to be admitted, in order to account for 
categorization $\mathrm{CP}$ requires an account of categories. The notion of $\mathrm{CP}$ not only postpones the problem it was supposed to solve, but using CP to account for categorization is circular to some extent. Nevertheless, categorical perception, or the enhanced discrimination behavior linked to categories remains an interesting phenomenon in and of itself. It requires to be explained. My final point was that the generally accepted naming strategy account was not enough to account for CP. Beyond labels, what seems to play a role in $\mathrm{CP}$ is category structure.

\section{References}

Bornstein, M. H., Kessen, W., \& Weiskopf, S. (1976). The categories of hue in infancy. Science, 191(201-202).

Bornstein, M. H. \& Korda, N. O. (1984). Discrimination and matching within and between hues measured by reaction times: some implications for categorical perception and levels of information processing. Psychological Research, 46, 207-222.

Daoutis, C., Pilling, M., \& Davies, I. (2006). Categorical effects in visual search of colour. Visual cognition, 14(217-240).

Davidoff, J., Goldstein, J., \& Roberson, D. (2009). Nature versus nurture: The simple contrast. Journal of Experimental Child Psychology, 102, $246-250$.

Davies, I. R. L. \& Corbett, G. (1998). A Cross-Cultural Study of ColorGrouping: Tests of the Perceptual-Physiology Account of Color Universals. Ethos, 26(3), 338-360.

Drivonikou, G. V., Kay, P., Regier, T., Ivry, R. B., Gilbert, A. L., Franklin, A., \& Davies, I. R. L. (2007). Further evidence that Whorfian effects are stronger in the right visual field than the left. PNAS, 104(3), 1097-1102.

Franklin, A., Clifford, A., Williamson, E., \& Davies, I. R. L. (2005). Color term knowledge does not affect categorical perception in toddlers. Journal of Experimental Child Psychology, 90, 114-141.

Franklin, A. \& Davies, I. R. L. (2004). New evidence for infant color categories. British Journal of Developmental Psychology, 22, 349-377.

Franklin, A., Drivonikou, G. V., Clifford, A., Kay, P., Regier, T., \& Davies, I. R. L. (2008a). Lateralization of categorical perception of color changes with color term acquisition. PNAS, 105, 18221-18225.

Franklin, A., Drivonikou, G. V., Bevis, L., Davies, I. R. L., Kay, P., \& Regier, T. (2008b). Categorical perception of color is lateralized to the right hemisphere in infants, but to the left hemisphere in adults. PNAS, 105, 3221-3225.

Gilbert, A., Regier, T., Kay, P., \& Ivry, R. (2006). Whorf hypothesis is supported in the right visual field but not the left. PNAS, 103(2), 489-494.

Hanley, J. R. \& Roberson, D. (2011). Categorical perception effects reflect differences in typicality on within-category trials. Psychonomic Bulletin \& Review, 18(2), 355-363.

Harnad, S., (1987). Psychophysical and cognitive aspects of categorical perception: A critical overview. In Harnad, S. (Ed) Categorical Perception: The Groundwaork of Cognition, Cambridge University Press, 1-28 
Hering, E. (1964). Outlines of a theory of the light sense. Cambridge, Massachussetts: Harvard University Press.

Jraissati, Y., Wakui, E., Decock, L., \& Douven, I. (2012). Constraints on colour category formation. International Studies in the Philosophy of Science (to appear).

Karni, A. \& Sagi, D. (1993). The time course of learning a visual skill. Nature, 365, 250-252.

Kay, P. \& Kempton, W. (1984). What is the Sapir-Whorf hypothesis?. American Anthropologist, 86, 65-79.

Kay, P. \& McDaniel, C. K. (1978). The linguistic significance of the meanings of basic color terms.. Language, 54(3), 610-646.

Liberman, A. M., Harris, K. S., Hoffman, H. S., \& Griffith, B. C. (1957). The discrimination of speech sounds within and across phoneme boundaries. Journal of Experimental Psychology, 54(358-368).

Massaro, D. \& Cohen, M. (1983). Categorical or continuous speech perception: a new test. Speech communication, 2, 15-35.

Massaro, D., (1987). Categorical partition: a fuzzy-logical model of categorization behavior. In Harnad, S. (Ed) Categorical Perception: The Groundwaork of Cognition, Cambridge University Press, 254-286

Özgen, E. \& Davies, I. R. L. (2002). Acquisition of Categorical Color Perception: A Perceptual Learning Approach to the Linguistic Relativity Hypothesis. Journal of Experimental Psychology: General, 131(4), 477493.

Pilling, M., Wiggestt, A., Özgen, E., \& Davies, I. R. L. (2003). Is color "categorical perception" really perceptual? Memory and Cognition, 31(4), 538-551.

Pisoni, D. B. \& Tash, J. (1974). Reaction times to comparisons within and across phonetic categories. Perception \& Psychophysics, 15(2), 285290.

Rensink, R. A. \& Enns, J. T. (1995). Preemption effects in visual search: Evidence for low-level grouping. Psychological Review, 102(1), 101-130.

Roberson, D. \& Davidoff, J. (2000). The categorical perception of colours and facial expressions: The effect of verbal interference. Memory \& Cognition, 28, 977-986.

Roberson, D., Davidoff, J., \& Braisby, N. (1999). Similarity and categorization : neuropsychological evidence for a dissociation in explicit categorization tasks. Cognition, 71(1), 1-42.

Roberson, D., Davies, I., \& Davidoff, J. (2000). Color categories are not universal : replications and new evidence from a Stone-Age culture. Journal of Experimental Psychology: General, 129(3), 369-398.

Roberson, D., Pak, H., \& Hanley, J. R. (2008). Categorical perception of colour in the left and right visual field is verbally mediated: Evidence from Korean. Cognition, 107(2), 752-762.

Rosch, E. (1973). Natural categories. Cognitive psychology, 4 (3), 328-350.

Schouten, B., Gerrits, E., \& van Hessen, A. (2003). The end of categorical perception as we know it. Speech communication, 41, 71-80.

Siok, W. T., Kay, P., Wang, S. Y. W., Chan, A. H. D., Chen, L., Luke, K.-K., \& Tan, L. H. (2009). Language regions of brain are operative in color perception. PNAS, 106, 8140-8145. 
Studdert-Kennedy, M., Liberman, A. M., Harris, K. S., \& Cooper, F. S. (1970). Motor theory of speech perception: a reply to Lane's critical review. Psychological Review, 77(3), 234-249.

Tan, A. H. D., Chan, L. H., Khong, P. L., Yip, L. K. C., \& Luke, K.-K. (2008). Language affects patterns of brain activation associated with perceptual decision. PNAS, 105(10), 4004-4009.

Whorf, B.-L. (1956). Language, thought and reality: selected writings. New York: John Wiley \& Sons.

Winawer, J., Witthoft, N., Frank, M. C., Wu, L., Wade, A. R., \& Boroditsky, L. (2007). Russian blues reveal effects of language on color discrimination. PNAS, 104, 7780-7785.

Wood, C. C. (1974). Parallel processing of auditory and phonetic information in speech perception. Perception \& Psychophysics, 15, 501-508.

Zhou, K., Mo, L., Kay, P., Kwok, V. P. Y., Ip, T. N. M., \& Tan, L. H. (2010). Newly trained lexical categories produce laterialized categorical perception of color. PNAS, 107(22), 9974-9978. 